\begin{tabular}{|c|l|}
\hline Title & Transient grating studies of phase and spin relaxations of excitons in GaA s single quantum wells \\
\hline Author(s) & Tomoda, K.; A dachi, S.; Muto, S.; Shimomura, S. \\
\hline Citation & $\begin{array}{l}\text { Physica E: Low-dimensional Systems and Nanostructures, 42(10), 2714.2717 } \\
\text { https://doi.org/10.1016/.physe.2009.12.009 }\end{array}$ \\
\hline Issue Date & 2010-09 \\
\hline Doc URL & http://hdl.handle.net/2115/50055 \\
\hline Type & article(author version) \\
\hline File Information & PhyE42-10_2714_2717.pdf \\
\hline
\end{tabular}

Instructions for use 


\title{
Transient grating studies of phase and spin relaxations of excitons in GaAs single quantum wells
}

\author{
K. Tomoda ${ }^{a}$, S. Adachi ${ }^{* a}$, S. Muto ${ }^{\text {a }}$, S. Shimomura ${ }^{\mathrm{b}}$ \\ ${ }^{a}$ Department of Applied Physics, Hokkaido University, Sapporo 060-8623, Japan \\ ${ }^{b}$ Graduate School of Science and Engineering, Ehime University, Matsuyama 790-8577, \\ Japan
}

\begin{abstract}
Exciton spin and phase relaxations at low temperatures in GaAs/AlGaAs single quantum wells were investigated by using transient grating technique. The technique allows us to obtain the exciton lifetime, spin relaxation, and phase relaxation in the same setup. In combination with a series of single quantum wells grown on the same substrate, the well width dependence of exciton spin relaxation was studied. The obtained spin relaxation results were analyzed with their phase relaxation times in a framework of MAS mechanism, and were in good agreement with the calculated results. Especially, the motional narrowing character of the exciton spin relaxation was well demonstrated.
\end{abstract}

Key words: exciton spin relaxation, phase relaxation, exchange interaction, GaAs, quantum well

\footnotetext{
*phone/fax: +81-11-706-6669

Email address: adachi-s@eng.hokudai.ac.jp (S. Adachi)
} 


\section{Introduction}

The spin relaxation processes of excitons and electrons in semiconductor nanostructures have been a central topic of intense experimental and theoretical investigations. Spin-based applications such as quantum information processing boost up the studies of spin coherence, especially, in quantum dots since quantitative information on spin relaxation or spin lifetime is essential to the design and successful implementation of devices.

As in bulk semiconductors, in the quantum well (QW), a conduction band electron and a valence band hole can bind into an exciton due to Coulomb attraction. However the exciton states are strongly modified by the confinement of electrons and holes in one direction. Because of the increased wavefunction overlap and the ability of exciton to acquire appreciable momentum during its long lifetime, the long-range exchange interaction has larger importance for spin relaxation process in type I QW than in bulk materials. Based on the studies since around 1990, exciton spin relaxation mechanism effective for GaAs QWs got settled; for room temperature, the scattering during the spin precession in the effective magnetic field originating from the spin splitting in conduction band, and for low temperatures, the exchange interaction between electron and hole in an exciton. The former is the D'yakonov-Perel'(DP) mechanism (1) and the latter is the QW version (Maialle-Andrada-Sham (MAS) mechanism (2)) of the Bir-Aronov-Pikus (BAP) mechanism (1) for electron spin relaxation in bulk semiconductors, respectively. However, there exists only a few reports on the well width dependence of spin relaxation, which corresponds to change the wavefunction overlap and allows one to deduce the dominant spin relaxation mechanism. 
Furthermore, in most of their reports, phase relaxation has been assumed as a constant regardless of the well width $(3 ; 4 ; 5 ; 6)$.

In the present work, the exciton spin and phase relaxations at low temperatures in GaAs/AlGaAs single quantum wells (SQWs) were studied by using transient grating technique. This technique allows us to obtain the exciton lifetime, spin relaxation, and phase relaxation for resonant condition in the same setup. The character that spin coherence depends strongly on the scattering events gives rise to a sample-to-sample distribution of the experimental spin relaxation times. The extent of confinement also should affect significantly to the spin coherence through the change of scattering. Therefore, the used technique gives a complete set of the information about spin relaxation dynamics.

\section{Sample and Experimental Setup}

The sample used here is GaAs/ $\mathrm{Al}_{0.3} \mathrm{Ga}_{0.7} \mathrm{As}$ SQWs. A series of SQWs with different well width separated by 200 - $\AA$-thick AlGaAs layers were grown on a (001) GaAs substrate by molecular beam epitaxy. The sample was mounted in a cold finger cryostat and was kept at $10 \mathrm{~K}$ through the experiments. Important basic values obtained by the steady state photoluminescence (PL) and PL excitation (PLE) measurements are listed in the upper part of Table 1.

In order to observe the transient processes, two-pulse and three-pulse fourwave-mixing (FWM) measurements in reflection geometry were performed by using a mode-locked Ti:sapphire laser with a repetition rate of $76 \mathrm{MHz}$. In the tuning range of $750-810 \mathrm{~nm}$, the laser bandwidth was $\sim 8.5 \mathrm{meV}$ (pulse 
width $\sim 150 \mathrm{fs}$ ). For spin relaxation and exciton lifetime measurements, two excitation pulses with orthogonally linear and parallel linear polarizations, respectively, are overlapped spatially and temporally on the sample. As a result, the respective excitation polarizations produce the exciton spin density grating $(\mathrm{SG})$ and the exciton density grating (DG) in the sample $(7 ; 8)$. The probe pulse with a controlled delay time is diffracted in backward direction by the created transient grating and can monitor the decay by the diffracted intensity. The decay rate of the respective transient grating $1 / \tau_{\mathrm{DG}(\mathrm{SG})}$ is given as $1 / \tau_{\mathrm{DG}(\mathrm{SG})}=1 / \tau_{\mathrm{R}(\mathrm{S})}+4 \pi^{2} D_{\mathrm{R}(\mathrm{S})} / \Lambda^{2}$, which has two components; exciton recombination (spin relaxation) and exciton diffusion (exciton spin diffusion) in exciton DG (exciton $\mathrm{SG}$ ). Here, $\tau_{\mathrm{R}(\mathrm{S})}$ is exciton recombination time (exciton spin relaxation time), $D_{\mathrm{R}(\mathrm{S})}$ is diffusion coefficient of exciton density (exciton spin density), and $\Lambda$ is the grating fringe spacing. In this measurements, $\Lambda$ is rather wide $(\sim 20 \mu \mathrm{m})$ due to the small crossing angle of the excitation pulse pair. Considering the diffusion coefficient in GaAs QW $\left(D_{\mathrm{R}} \sim 16.5 \mathrm{~cm}^{2} / \mathrm{s}, D_{\mathrm{S}} \sim 3.5 D_{\mathrm{R}}\right)(8)$, contribution of diffusion effects to the grating decay is negligible in the observed time scale. The details of the spin grating technique are seen in Refs. $(7 ; 8 ; 9 ; 10)$. For phase relaxation measurements, one of three pulses was blocked and only two of three pulses were used to generate and monitor the exciton polarization grating $(\mathrm{PG})$. The self-diffracted FWM signals were spectrally-resolved or spectrally-integrated for the proper purpose. In all FWM measurements, the laser wavelength was tuned to the heavy hole exciton $(\mathrm{XH})$ resonance. 
Table 1: Important values of the used SQWs. $L_{\mathrm{W}}, E_{\mathrm{XH}}, \Delta E_{\mathrm{XL}-\mathrm{XH}}$, and $E_{\mathrm{XH}}^{\mathrm{b}}$ represent the well thickness, heavy hole exciton energy $(10 \mathrm{~K})$, splitting energy between heavy hole and light hole excitons $(10 \mathrm{~K})$, and binding energy of heavy hole exciton, respectively. Also, the obtained values of phase relaxation time $\tau_{\mathrm{m}}$, exciton spin relaxation time $\tau_{\mathrm{S}}$, and exciton lifetime $\tau_{\mathrm{R}}$ at $10 \mathrm{~K}$ in the present experiments are shown in the lower part.

\begin{tabular}{c|c|c|c|c} 
QW sample & $\mathrm{A}$ & $\mathrm{B}$ & $\mathrm{C}$ & $\mathrm{D}$ \\
\hline$L_{\mathrm{W}}(\AA)$ & 120 & 72 & 48 & 36 \\
$E_{\mathrm{XH}}(\mathrm{eV})$ & 1.5368 & 1.5669 & 1.6069 & 1.6448 \\
$\Delta E_{\mathrm{XL}-\mathrm{XH}}(\mathrm{meV})$ & 6.8 & 15.4 & 23.7 & 29.4 \\
$E_{\mathrm{XH}}^{\mathrm{b}}(\mathrm{meV})$ & 9.0 & 10.8 & 12.2 & 13.0 \\
\hline$\tau_{\mathrm{m}}(\mathrm{ps})$ & 7.9 & 1.8 & 3.6 & 6.2 \\
$\tau_{\mathrm{S}}(\mathrm{ps})$ & 25.0 & 68.2 & 23.0 & 17.0 \\
$\tau_{\mathrm{R}}(\mathrm{ps})$ & 730 & 230 & 380 & 270 \\
\hline
\end{tabular}

\section{Results and Discussion}

As an example of the decay of the respective transient grating, we show the time-integrated FWM signals in $120-\AA$ and 48 - $\AA$ SQWs at $10 \mathrm{~K}$ in Fig. 1. From the uppermost panel, exciton phase relaxation (two-pulse FWM with PG), exciton spin relaxation (three-pulse FWM with SG), and exciton recombination (three-pulse FWM with DG) are represented.

\subsection{Phase Relaxation}

Phase relaxation of the exciton polarization occurs by the scattering mainly by phonon and other excitons in large well width, and corresponds to the momentum relaxation of the center of mass motion of excitons. It 

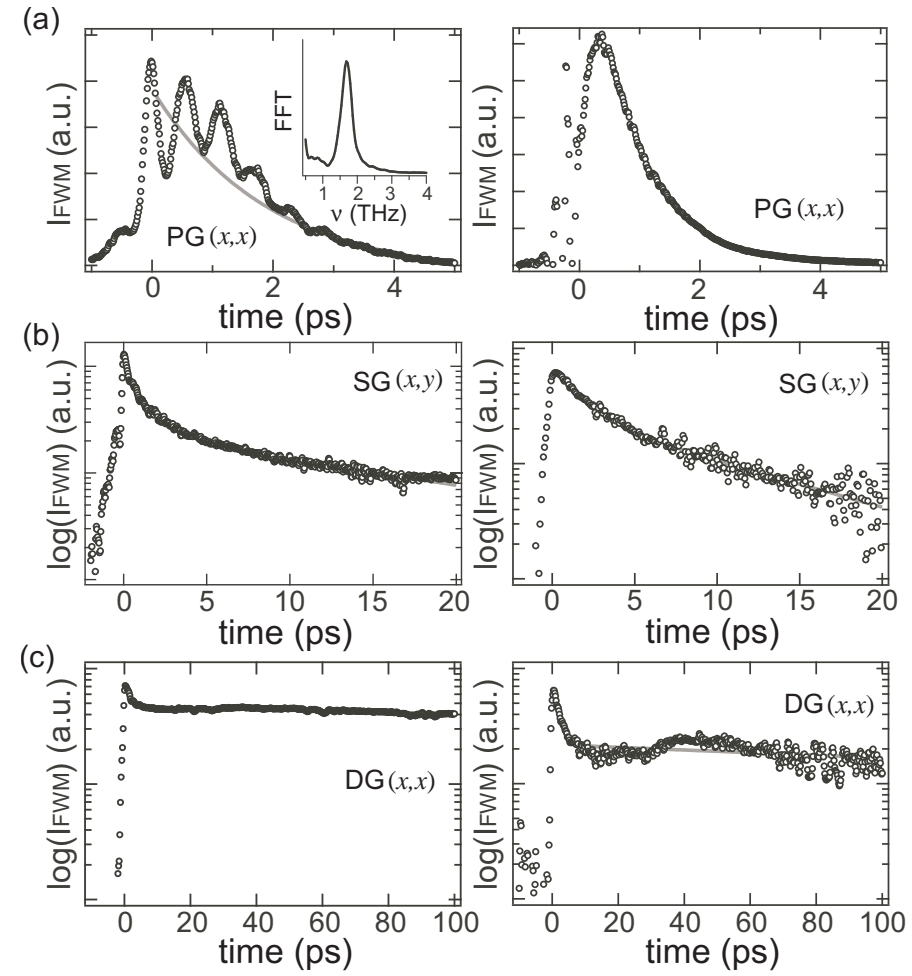

Figure 1: Typical signals of two-pulse and three-pulse FWM measurements in 120- $\mathrm{A}$ SQW (left column) and $48-\AA$ SQW (right column) at $10 \mathrm{~K}$. (a) Phase relaxation of excitons monitoring the decay of polarization grating (PG) by two-pulse FWM. inset (left column): Fourier transformed spectrum of the signal. The peak due to the quantum beat is found to be $\sim 1.64 \mathrm{THz}(6.8 \mathrm{meV})$. (b) Exciton Spin relaxation monitoring the decay of spin density grating (SG) by three-pulse FWM. (c) Exciton lifetime monitoring the decay of exciton density grating (DG) by three-pulse FWM. In (a) of the right column, the spiky signal around the time origin is due to interference of two pulses and has no influence to obtain the phase relaxation time.

can be monitored by the decay of exciton polarization grating, which can be produced by the second pulse incoming within the time interval until the coherently created exciton polarization by the first pulse relaxes completely. Here, as an example of phase relaxation, let us consider it in a $120-\AA \mathrm{SQW}$. Since $120-\AA$ SQW has the comparable XL-XH splitting energy $\Delta E_{\mathrm{XL}-\mathrm{XH}}$ 
(see Table 1) to the laser bandwidth, the simultaneously excited XH and $\mathrm{XL}$ gives rise to the beating in the FWM signal. The beat period is $\sim 0.61$ ps $(1.64 \mathrm{THz})$ and coincides with the $\Delta E_{\mathrm{XL}-\mathrm{XH}}$. This agreement and the spectrally-resolved measurements (not shown here) indicate that the beat occurs quantum mechanically between XL and XH states although the biexcitonic effect are also shown in negative delay time and around the first peak. Duration of the beating and the fundamental decay are determined by the phase relaxation of XL and XH, respectively, because XL has shorter phase relaxation than $\mathrm{XH}$.

Within a framework of optical Bloch equation for $\delta$-function pulses, the FWM signal due to the third-order nonlinearity decays single exponentially in the form of $I_{\mathrm{FWM}} \propto I_{0} \exp \left(-c \tau / \tau_{\mathrm{m}}\right)$. The constant $c$ is given as 2 (4) at the limit of a purely homogeneously (highly inhomogeneously) broadened two-level system. For intermediate case, the signal decays biexponentially whose decay rate is initially $2 / \tau_{\mathrm{m}}$ and subsequently changes to $4 / \tau_{\mathrm{m}}$. Considering the decay time $(\sim 2 \mathrm{ps})$ in the long time region compared with its spectral width, the XH line was found to be inhomogeneously broadened. Consequently, the phase relaxation time $\tau_{\mathrm{m}}$ of $\mathrm{XH}$ is deduced to $7.9 \mathrm{ps}$. The FWM signals in other three SQWs has no quantum beat and the obtained $\tau_{\mathrm{m}}$ is listed in Table 1. As seen in the table, the SQW with the well width of $72 \stackrel{\circ}{A}$ shows the short phase relaxation and exciton lifetime out of the overall dependence. Since the same growth condition was used for all QSWs, the scatterings by impurities and phonon are supposed to be efficient for all SQWs to the same extent. We suppose tentatively the SQW has larger number of the scattering centers originate from the interface compared to other 
QWs, though no clear evidence of the dominant cause for the well width dependence is obtained at present.

\subsection{Spin Relaxation}

The middle and bottom panels of Fig. 1 show the three-pulse FWM signals for $(x, y)$ and $(x, x)$ polarizations of the excitation pulse pair, whose polarization combination creates the $\mathrm{SG}$ and $\mathrm{DG}$, respectively. Remember the decays of SG and DG represent the exciton spin relaxation and exciton recombination, respectively, and note that the time scales of those signals are very different. However, in the middle panel, the obtained signals indicate the apparent multiexponential decay. The faster decay in the range of 0-3 ps consists of the following components; (1) coherent artifact, (2) spin relaxation of hole in an exciton (9), and (3) phase relaxation signal due to one of the excitation pulse pair and the probe pulse (11). Those faster decay components are not problems because the time scale is very different from the slower decay component that represents the exciton spin relaxation. In addition, because of resonant excitation and low temperature, direct relaxation with mutual electron hole spin-flip due to the exchange interaction becomes dominant compared with the indirect one with sequential spin-flips of the single particles (i.e. population transfer to dark exciton states). Therefore, we can deduce safely the exciton spin relaxation time and the exciton recombination time for the respective grating. Assuming the population transfer to the dark exciton states is negligible, the slower decay component of the SG and DG can be written by $I_{\mathrm{FWM}}^{\mathrm{SG}}(t) \propto \exp \left[-\left(2 / \tau_{\mathrm{S}}+1 / \tau_{\mathrm{R}}\right) t\right]$ and $I_{\mathrm{FWM}}^{\mathrm{DG}}(t) \propto \exp \left(-t / \tau_{\mathrm{R}}\right)$, respectively, from the rate equations in bright-dark exciton system (9). Since $\tau_{\mathrm{R}} \gg \tau_{\mathrm{S}}$ as shown in Fig. 1, the influence of exciton recombination is very 


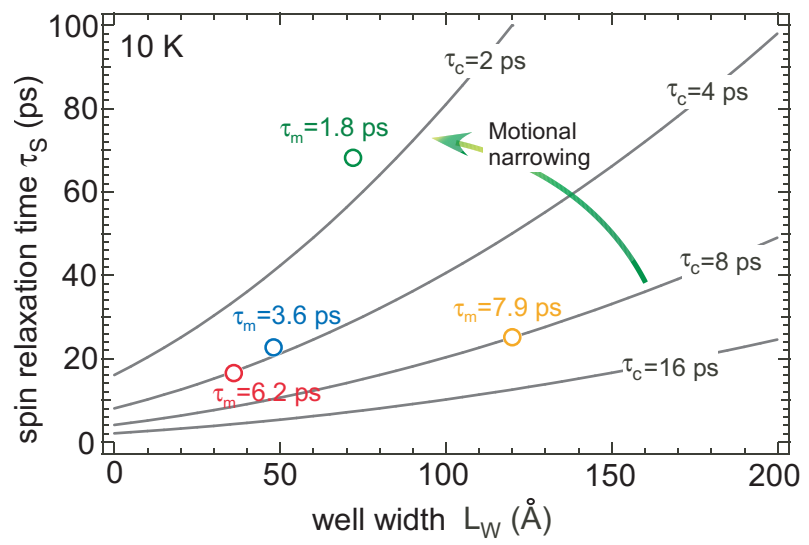

Figure 2: Spin relaxation times of four single QWs with different well width are plotted. The phase relaxation time $\tau_{\mathrm{m}}$ of their QWs are also indicated. The solid curves are the theoretical prediction considering the exchange interaction

small in our case and the exciton spin relaxation time becomes nearly twice the decay time of the FWM signals in SG.

Spin relaxation can be understood generally as a result of the action of randomly fluctuating magnetic field (RFM field). The spin makes a precession around the random direction of this effective RFM field with a typical frequency $\Omega$ and during a typical time $\tau_{\mathrm{c}}$. This characteristic time $\tau_{\mathrm{c}}$ is called a correlation time, which means the time during which the field may be roughly considered as constant. After a time $\tau_{\mathrm{c}}$ the direction and the field amplitude change randomly, and the spin starts its precession around the new direction of the field. After a certain number of such steps the initial spin direction will be reduced.

In the case that the precession angle for a single step $\Omega \tau_{\mathrm{c}} \ll 1$, the spin vector experiences a slow angle diffusion. After a time $t$, since number of the random steps is $t / \tau_{c}$, the averaged squared precession angle is $\left\langle\left(\Omega \tau_{\mathrm{c}}\right)^{2}\right\rangle t / \tau_{\mathrm{c}}$. 
The spin relaxation time $\tau_{\mathrm{s}}$ is defined as the time at which this angle becomes $\sim 1$, i.e. $\left\langle\left(\Omega \tau_{\mathrm{c}}\right)^{2}\right\rangle \tau_{\mathrm{s}} / \tau_{\mathrm{c}} \sim 1$. Consequently, $\tau_{\mathrm{s}}$ is given as $\tau_{\mathrm{s}} \sim 1 /\left(\Omega^{2} \tau_{\mathrm{c}}\right)$. That means that spin relaxation at the condition $\Omega \tau_{\mathrm{c}} \ll 1$ shows the motional narrowing. Maialle, de andrada e Silva, and Sham developed the theory for the exciton spin relaxation mechanism due to electron-hole exchange Coulomb interaction in QWs (MAS mechanism) (2). In this mechanism, the spin-flip process can be associated with the RFM field in the well interface plane, which originates in the electron-hole exchange, and the magnitude and direction of the RFM field depend on the exciton center of mass (COM) momentum $K$. Since the fluctuations due to the scattering of COM momentum is responsible for the exciton spin relaxation, the correlation time $\tau_{\mathrm{c}}$ in the mechanism is considered to be the COM momentum relaxation time $\tau_{\mathrm{m}}$.

Figure 2 shows the experimental values of exciton spin relaxation in four SQWs with $\tau_{\mathrm{m}}$ which is obtained by two-pulse FWM measurements (the uppermost panels of Fig. 1). The solid lines represent the calculated well width dependence of exciton spin relaxation time for different values of the correlation time $\tau_{\mathrm{c}}$. In the calculation, we assumed the GaAs QW with infinite barrier and exciton COM kinetic energy $E_{\mathrm{K}}$ of $0.4 \mathrm{meV}$. Other parameters such as an effective mass and Luttinger parameters are the same as those in Ref. (2). The obtained data agree well with the calculated results at $E_{\mathrm{K}}=0.4 \mathrm{meV}$ assuming $\tau_{\mathrm{c}}=\tau_{\mathrm{m}}$. We adopted this value of $E_{\mathrm{K}}$ as a fitting parameter and it is approximately a mean $\Gamma_{\mathrm{h}}$ of the investigated SQWs. Maialle et al. assumes $E_{\mathrm{K}}$ approximately equal to the homogeneous broadening $\Gamma_{\mathrm{h}}$ of the exciton states $(2)$. Since $\Gamma_{\mathrm{h}}$ has the relation to the COM momentum relaxation time $\tau_{\mathrm{m}}$ by $\Gamma_{\mathrm{h}}=\hbar /\left(\pi \tau_{\mathrm{m}}\right)$, their assumption leads to 
$E_{\mathrm{K}} \tau_{\mathrm{m}} \simeq \Gamma_{\mathrm{h}} \tau_{\mathrm{m}}=$ constant. In the used SQWs in this experiments, the value of $\Gamma_{\mathrm{h}}$ is ranging from 0.16 to $0.73 \mathrm{meV}$ from the measured $\tau_{\mathrm{m}}$. The assumption $E_{\mathrm{K}} \simeq \Gamma_{\mathrm{h}}$ asks $E_{\mathrm{K}}$ to change in conjunction with the change of $\tau_{\mathrm{m}}$, and cosequently the experimental data fall in a single curve by calculation, as seen in Ref. 2. However, our results suggests that the $E_{\mathrm{K}}$ is determined independently of $\Gamma_{\mathrm{h}}$, since exciton spin relaxation in different QWs were reproduced by single value of $E_{\mathrm{K}}$. At this point, further detailed quantitative analysis is necessary including the physical origin of the well-width dependence of phase relaxation time. Anyway, overall feature can be explained by MAS mechanism, and especially the motional narrowing of the exciton spin relaxation in these SQWs are clearly demonstrated.

\section{Summary}

We studied the exciton phase and spin relaxations in GaAs/AlGaAs SQWs at $10 \mathrm{~K}$ by using the transient grating technique. The character that spin coherence depends strongly on the scattering events gives rise to a sample-to-sample distribution of the experimental spin relaxation times. Therefore, both measurements of phase and spin relaxation times gave the enough information of exciton spin dynamics. The observed spin relaxation was explained well by MAS mechanism, and especially the motional narrowing behavior was clearly demonstrated. 


\section{References}

[1] F. Meier and B. Zakharchenya (eds.) Optical Orientation, Modern Problems in Condensed Matter Sciences Vol. 8 (North-Holland, NewYork, 1984).

[2] M. Z. Maialle, E. A. de Andrada e Silva, and L. J. Sham, Phys. Rev. B 47, $15776(1993)$.

[3] A. Vinattieri, J. Shah, T. C. Damen et al., Phys. Rev. B 50, 10868 (1994).

[4] P. Perozzo and A. Miller, Proc.of SPIE 2694, 51 (1996).

[5] A. Tackeuchi, Y. Nishikawa, and O. Wada, Appl. Phys. Lett. 68, 797 (1996).

[6] A. Malinowski, R. S. Britton, T. Grevatt et al., Phys. Rev. B 62, 13034 (2000).

[7] A. R. Cameron, P. Riblet, and A. Miller, Phys. Rev. Lett. 76, 4793 (1996).

[8] S. Adachi, Y. Takagi, J. Takeda, and K. A. Nelson, Opt. Commun. 174, $291(2000)$.

[9] O. Ikeuchi, S. Adachi, H. Sasakura, and S. Muto, J. Appl. Phys. 93, 9634 (2003).

[10] T. Watanuki, S. Adachi, H. Sasakura, S. Muto, Applied Phys. Lett. 86, 063114 (2005). 
[11] In principle, phase relaxation signal should not come into the same direction as the spin relaxation for $\mathrm{SG}$ and the exciton recombination for DG. However, the small intersection angle of the three pulses makes the small separation angle between two-pulse and three-pulse FWM signals. As a result, a part of two-pulse self-diffracted FWM signal was detected with the true three-pulse FWM signal in this experiments. 